\title{
Editorial
}

\section{Vitamin A as adjunct therapy for cerebral malaria: what is the evidence?}

\author{
*Achan J, Mpimbaza A \\ Department of Pediatrics and Child Health, Makerere University College of Health Sciences \\ http://dx.doi.org/10.4314/ahs.v12i2.1
}

Severe malaria continues to cause significant morbidity and mortality in several settings in Africa despite the availability of efficacious and potent antimalarial drugs. This has led to an increased interest in adjunctive therapies that target the underlying pathophysiology of severe malaria as additional approaches to management that may further reduce morbidity and mortality. Several adjunctive therapies have been tested over the years including dexamethasone, exchange blood transfusion, Nacetylsysteine, anti-TNF, mannitol and levamisole. However despite numerous clinical trials, to date, effective adjunctive treatments have been elusive. Most trials have been underpowered and in a recent review many were found to have inadequate methodological quality.

It is apparent therefore that different approaches to adjunctive therapy for severe malaria have been evaluated. One additional approach has been exploring the utilization of micronutrients in the management of malaria. Whereas micronutrients are well known to have antioxidant effects, their interaction with malaria is not well characterized. Their role for the treatment and prevention of malaria has however been a source of intrigue with studies reporting conflicting results on their impact. Zinc, vitamin $A$ and $E$, carotenoids, vitamins $B$ and $C$ and folic acid have been reported to have modulatory effects on the pathogenesis of malaria ${ }^{1}$. Beneficial protective effects of vitamin A or zinc on malariarelated morbidity have been demonstrated in some settings ${ }^{2,3,4,5}$. In Burkina Faso, combined supplementation with vitamin $\mathrm{A}$ and zinc reduced

\begin{tabular}{|l|}
\hline *Correspondence author: \\
Jane Achan \\
Department of Paediatrics and Child Health \\
Makerere University, College of Health Sciences \\
P.O. Box 7072 \\
Mulago Hill Road \\
Kampala, Uganda \\
Email: achanj@yahoo.co.uk
\end{tabular}

African Health Sciences Vol 12 No 2 June 2012 the burden of clinical attacks of malaria in young children by $30 \% 0^{6}$. In contrast to these studies that have suggested potential protective effects of vitamin A or zinc supplementation, other studies have shown the reverse. A randomized, placebo-controlled trial in Burkina Faso of zinc supplementation failed to show a protective impact on malaria episodes ${ }^{7}$. In addition, a study done in 2006 found that iron and folic acid supplements instead increased malariarelated hospitalization and deaths by 12 per cent. In a more recent study in Tanzania, giving supplements containing iron, folic acid, copper and vitamins to about 600 children aged 6 months to 5 years with iron deficiency increased their likelihood of contracting malaria by 41 per cent ${ }^{8}$. These conflicting findings indicate that currently, there is no consensus on the exact impact of micronutrients on malaria treatment and prevention.

In this issue of the African Health Sciences, Mwanga et al report results of a randomized controlled trial evaluating the effect of vitamin A adjunct therapy for cerebral malaria in children admitted to Mulago hospital' ${ }^{9}$. As a leading cause of childhood mortality in sub-Saharan Africa, interventions to ameliorate mortality due to cerebral malaria are a public health priority. Vitamin A is an essential nutrient required for maintaining immune function, playing an important role in the regulation of cell-mediated immunity and in humoral antibody responses. Vitamin A has been shown to suppress growth of P.falciparum, scavenge free radicals, and exhibit synergistic action with quinine in parasite clearance $^{10,10}$. Mwanga et al studied 142 children aged 6 months to 5 years, who were randomized to receive either Vitamin A or placebo and antimalarial treatment with intravenous quinine. The results show that whereas there were no significant differences in outcome measures between the two treatment arms, there was a trend towards earlier resolution of convulsions and lower mortality $(8.1 \%)$ in children who received Vitamin A supplementation compared 
to $16.2 \%$ in the placebo. Whereas this difference in mortality did not reach statistical significance; it draws attention to the potential role of Vitamin $\mathrm{A}$ as adjunct therapy for cerebral malaria. Vitamin A is a particularly attractive option for this role as it is a relatively affordable, easy to administer and readily available intervention that could be appropriate for use in several malaria endemic countries. However, before any such recommendations are made, a sufficiently powered study with different dosing regimens are warranted to provide more conclusive evidence for the role Vitamin A could play as adjunct therapy. Furthermore reduction in severe malaria related mortality will also be achieved through embracing the use of intravenous artesunate as the preferred first line drug for the management of severe malaria. In several settings in sub-Saharan Africa, additional interventions will include improved health seeking behavior that will promote early treatment seeking, strengthening of referral systems, improved triage and emergency treatment approaches and finally better supportive care for hospitalized patients.

\section{References}

1. Okochi VI and Okpuzor J. Micronutrients as therapeutic tools in the management of sickle cell disease, malaria and diabetes. African Journal of Biotechnology 2005; 4 (13):.1568-1579, Dec.

2. Richard SA, Zavaleta N, Caulfield LE, Black RE, Witzig RS, Shankar AH. Zinc and iron supplementation and malaria, diarrhea, and respiratory infections in children in the Peruvian Amazon. Am J Trop Med Hyg. 2006; 75:126132.

3. Shankar AH, Genton B, Baisor M, Paino J, Tamja $\mathrm{S}$, Adiguma T, Wu L, et al. The influence of zinc supplementation on morbidity due to Plasmodium falciparum: a randomized trial in preschool children in Papua New Guinea. Am J Trop Med Hyg. 2000; 62:663-669.

4. Shankar AH, Genton B, Semba RD, Baisor M, Paino J, Tamja S, Adiguma T, Wu L, et al. Effect of vitamin A supplementation on morbidity due to Plasmodium falciparum in young children in Papua New Guinea: a randomised trial. Lancet. 1999; 354:203-209.

5. Cusick SE, Tielsch JM, Ramsan M, Jape JK, Sazawal S, Black RE, Stoltzfus RJ. Short-term effects of vitamin $A$ and antimalarial treatment on erythropoiesis in severely anemic Zanzibari preschool children. Am J Clin Nutr. 2005; 82:406-412.

6. Zeba AN, Sorgho H , Rouamba N, Zongo I, Rouamba J, Guiguemdé TR, Hamer HD et al. Major reduction of malaria morbidity with combined vitamin A and zinc supplementation in young children in Burkina Faso: a randomized double blind trial. Nutr J. 2008; 7: 7.

7. Binka F, Ross D, Morris S. Vitamin A supplementation and childhood malaria in northern Ghana. Am J Clin Nutr. 1995; 61:853859.

8. Veenemans J, Milligan P, Prentice MA, Laura RA. Schouten NI, van der Heijden AC, Linsey C, et al. Effect of Supplementation with Zinc and Other Micronutrients on Malaria in Tanzanian Children: A Randomized Trial. PLoS Med 8(11): e1001125. doi:10.1371/journal.pmed.1001125

9. Mwanga-Amunpaire J, Ndeezi G, Tumwine JK Effect of vitamin A adjunct therapy for cerebral malaria in children admitted to Mulago hospital: a randomized controlled trial. African Health Sciences 2012; 12(2): 90 - 97.

10. Serghides L, Kain KC. Mechanism of protection induced by vitamin A in falciparum malaria. Lancet 2002; 359:1404-06

11. Skinner AT, Barrett H. Heterogenous activity in vitro of Vitamin A (retinol) in combination with novel established antimalarial drugs. Trans $\mathrm{R}$ Soc Trop Med Hyg. 1999; 93(5): 550-1. 